\title{
Analysis of the Step Responses of Laminar Premixed Flames to Forcing by Non-thermal Plasma
}

\author{
Deanna A. Lacoste ${ }^{\mathrm{a}}$, Jonas P. Moeck ${ }^{\mathrm{b}}$, William L. Roberts ${ }^{\mathrm{a}}$, Suk Ho \\ Chung $^{\mathrm{a}}$, Min Suk Cha ${ }^{\mathrm{a}}$ \\ ${ }^{a}$ King Abdullah University of Science and Technology, Clean Combustion Research \\ Center, Thuwal 23955-6900, Saudi Arabia \\ ${ }^{b}$ Technische Universität Berlin, Institut für Strömungsmechanik und Technische Akustik, \\ 10623 Berlin, Germany
}

\begin{abstract}
The step responses of lean methane-air flames to non-thermal plasma forcing is reported. The experimental setup consists of an axisymmetric burner, with a nozzle made of a quartz tube. The equivalence ratio is 0.95, allowing stabilization of the flame in a V-shape or an M-shape geometry, over a central stainless steel rod. The plasma is produced by short pulses of 10-ns duration, 8-kV maximum voltage amplitude, applied at $10 \mathrm{kHz}$. The central rod is used as a cathode, while the anode is a stainless steel ring, fixed on the outer surface of the quartz tube. Plasma forcing is produced by positive or negative steps of plasma. The step response of the flame is investigated through heat release rate (HRR) fluctuations, to facilitate comparisons with flame response to acoustic perturbations. The chemiluminescence of $\mathrm{CH}^{*}$ between two consecutive pulses was recorded using an intensified camera equipped with an optical filter to estimate the HRR fluctuations. First, the results show that the flame does not respond to each single plasma pulse, but is affected only by the average plasma power, confirming the step nature
\end{abstract}


of the forcing. The temporal evolutions of HRR are analyzed and the flame transfer functions are determined. A forcing mechanism, as a local increase in the reactivity of the fluid close to the rod, is proposed and compared with numerical simulations. Experiments and numerical simulations are in good qualitative agreement.

Keywords: plasma-assisted combustion, flame dynamics, nanosecond repetitively pulsed glow discharges, flame transfer function, $G$-equation

\section{Introduction}

Thermoacoustic instabilities of a combustion front result from the interaction of unsteady heat release rate in a flame and the acoustic modes of a combustion chamber [1]. They can lead to high-amplitude oscillations of the pressure, the flow field, and the flame. Avoiding the occurrence of combustion instabilities is a major challenge in the design of modern stationary gas turbines and aeroengines [2]. Recently, the use of non-equilibrium plasma discharges to mitigate combustion instabilities has been studied [3-7]. In particular, nanosecond repetitively pulsed (NRP) discharges were successfully applied to turbulent premixed flame configurations to control thermoacoustic instabilities [3, 4]. Full suppression of instabilities could be obtained but, due to strongly coupled interaction mechanisms, detailed understanding of this effect is still lacking.

Generally, plasma discharges may act on the flame through thermal, chemical, and electric field effects, the latter affecting diffusive transport of ions as well as the hydrodynamic field [8-12]. In the particular case of NRP discharges, which are obtained for high values of the reduced electric 
field, the strong effect of the plasma on flame stability can only be attributed to coupled thermal and chemical activation of the fluid $[4,13]$, due to the nanosecond time scale of electric-field application. The effect of NRP discharges could then be considered as a local increase in the reactivity of the fluid.

To support this assertion and to evaluate the sensitivity of the flame response to a non-thermal plasma, this study presents the effect of NRP glow discharges, generated in the combustion area, on the dynamics of laminar methane-air premixed flames. This configuration has been chosen to sim-

plify as much as possible the plasma-flame coupling, although admittedly quite removed from industrial applications. Consequently, the effects of flow fluctuations, gaseous mixture inhomogeneities, distance between plasma and flame, or hydrodynamic effects of the plasma, are not investigated here. Instead, the step responses of laminar methane-air premixed flames to nonthermal plasma forcing are investigated. Based on the deduced flame transfer functions (FTFs), and on comparisons between experimental and simulated flame dynamics, the effect of NRP glow discharges, as a local increase in the reactivity of the fluid, is discussed.

\section{Experimental setup}

The experimental setup presented in Fig. 1 consists of a burner producing a laminar flame, an electric system generating NRP glow discharges, and diagnostics for plasma, flow and flame characterization. 


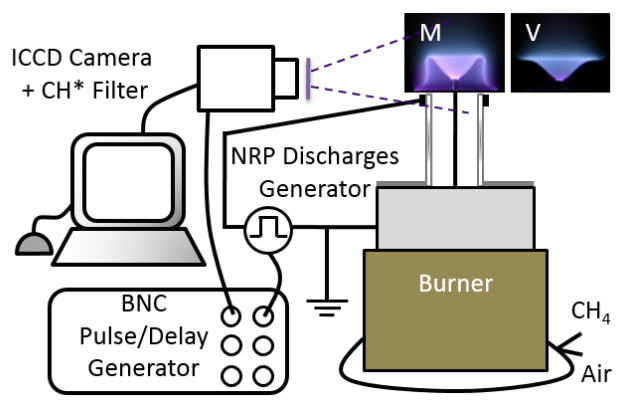

Figure 1: Schematic of the experimental setup.

\subsection{Laminar premixed burner}

An axisymmetric laminar burner, fed with a premixture of methane and air, is used. The flows of gases, controlled by mass flowmeters, are injected at the lowest part of a 120-cm long plenum. To minimize the boundary layer at the outlet of the injection tube, the flow passes through a nozzle, similar to that used by Mazas et al. [14], with a contraction ratio ratio of 86 . Thus, the internal profile of the burner allows a top-flat velocity profile at the outlet of the injection tube. This tube is made from quartz of 1-mm thickness and has an inner diameter of $7 \mathrm{~mm}$. A stainless steel rod with a diameter of 0.85 $\mathrm{mm}$ is centered inside the quartz tube and oversteps its outlet by $2 \mathrm{~mm}$. The equivalence ratio is 0.95 , allowing stabilization of the flame in a V-shape or an M-shape geometry. The flow rates of gases are chosen to obtain a thermal power released by the flame of $113 \mathrm{~W}$, corresponding to a bulk velocity of the flow at the outlet of the quartz tube of about four times the laminar burning velocity for these conditions. The bulk flow velocity at the outlet of the injection tube is $1.26 \mathrm{~ms}^{-1}$. The associated Reynolds number, based on the inner diameter of the quartz tube, is 400 . 


\subsection{Nanosecond repetitively pulsed discharge system}

Forcing by NRP discharges is generated with a solid-state pulse generator (FID Technologies FPG 10-30NM10). High voltage pulses have 10-ns duration, $10-\mathrm{kHz}$ repetition frequency, and up to $8-\mathrm{kV}$ amplitude. The stainless steel rod at the center of the burner is connected to the ground and serves as the cathode. The anode is a stainless steel ring located at the outlet of the quartz tube (see Figs. 1 and 2). It is important to note that, as the cathode protrudes the quartz tube by $2 \mathrm{~mm}$, and the anode is flush with its upper face, the quartz tube is not located in the interelectrode area. Therefore, no dielectric barrier discharges can be obtained. This can be verified in Fig. 2, where the plasma and the electrodes are visible, but not the quartz tube, located below the region of interest.

Energy deposited by the plasma is determined from the amplitude of the voltage and current pulses through the electrodes, measured with a high voltage probe (Tektronix P6015A), and a Pearson Coil (Model 6565). Data are recorded by an oscilloscope of $1 \mathrm{GHz}$ sampling rate (Lecroy Wavepro 7100A). The measurements are made with the flame present. Voltage and current signals are first synchronized. The voltage and conduction current waveforms (obtained after subtracting the capacitive current from the total current) are then multiplied and integrated to obtain the energy deposition per pulse [15]. The average power of the plasma is determined by multiplying the energy per pulse by the repetition frequency of the discharge. Plasma power depends strongly on applied voltage and thermal conditions between the electrodes. For the M-flame, when the applied voltage is $7 \mathrm{kV}$, the averaged power of the plasma, $P_{P}$, is $1 \mathrm{~W}$, while for an applied voltage of $8 \mathrm{kV}, P_{P}$ increases to 
$2.2 \mathrm{~W}$. For the $\mathrm{V}$-flame, when the applied voltage is $8 \mathrm{kV}, P_{P}$ is $1.7 \mathrm{~W}$. Current measurements also permit confirmation of the glow regime of the NRP discharges, i.e., low conduction current compared to the total current [16], and to assess the non-thermal nature of the plasma.

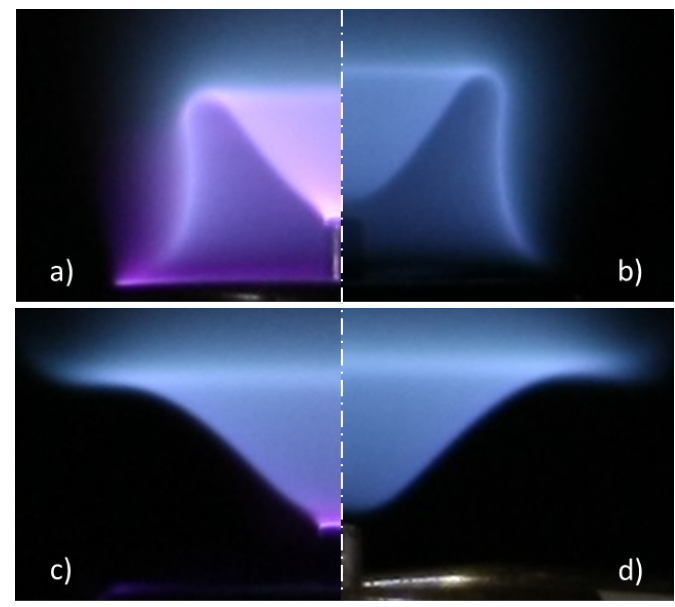

Figure 2: Photographs of the M- and V-flames, a) and c) with NRP glow discharges at $10 \mathrm{kHz}$ and 8-kV applied voltage, b) and d) without plasma (exposure time $0.8 \mathrm{~s}$ ).

To illustrate the effect of the NRP glow discharges on the M- and Vflames, Fig. 2 shows two combined photographs, a) and c) with NRP glow discharges at $10 \mathrm{kHz}$ and $8-\mathrm{kV}$ applied voltage, and b) and d) without plasma. These pictures clearly show that the plasma is located within the flame, and that the light intensity from the plasma is higher close to the cathode. It is also noticed that the flames enhanced by plasma are slightly more compact compared to the flames without plasma. Finally, plasma distribution is not similar for both geometries: the plasma is distributed more homogeneously into the M-flame than into the V-flame. 


\subsection{Diagnostics}

The measurements of $\mathrm{CH}^{*}$ chemiluminescence are made with a $1024 \times 1024$ pixel intensified-CCD camera (Princeton Instruments PIMAX3) equipped with a 10-nm bandpass filter (CVI Melles Griot F10-430.0-4-50.0M) centered at $430 \mathrm{~nm}$. For determination of the heat release rate (HRR) based on $\mathrm{CH}^{*}$ emission (see next section), the signal collection is gated and light emission of the flame, between the plasma pulses, is recorded. A delay generator (Berkeley Nucleonics BNC Model 575), synchronizes the FID pulse generator and the camera. To increase the signal-to-noise ratio, images are averaged over 200 events. After background subtraction, each image is spatially averaged to obtain the $\mathrm{CH}^{*}$ chemiluminescence intensity of the entire combustion area. The temporal evolution of the $\mathrm{CH}^{*}$ chemiluminescence is then determined and analyzed to obtain the flame transfer functions (FTFs).

\section{HRR experimental determination}

The spontaneous emission of excited methylidyne radicals $\left(\mathrm{CH}^{*}\right)$ is used to determine the HRR fluctuations of the flame. This technique has been validated for perfectly premixed flames $[17,18]$, and is extended here, according to previous studies $[11,4]$, for the determination of HRR fluctuations of a flame submitted to plasma forcing. However, in order to verify that the $\mathrm{CH}^{*}$ emission is not affected by the plasma, optical emission spectroscopy measurements were conducted in the wavelength range of the light collection, i.e., between 410 and $450 \mathrm{~nm}$. Figure 3a presents a comparison of the spectra obtained for the flame alone (black), and the flame enhanced by NRP

discharges (red). In the case of plasma forcing, because light emission from 
the plasma (mainly due to the $\mathrm{N}_{2}(\mathrm{C}-\mathrm{B})$ transition, in the wavelength range of interest), cannot be spatially separated from flame emission light (see Fig. 2), the spectrum should be recorded between two plasma pulses. This requirement fixed the acquisition settings, kept identical for the two spectra presented on Fig. 3a, i.e., with an integration time of $98 \mu$ s. The two spectra obtained, with and without plasma enhancement, are identical. Therefore, for similar acquisition conditions, the $\mathrm{CH}^{*}$ chemiluminescence of the flame is used to determine the HRR fluctuations with and without plasma forcing.
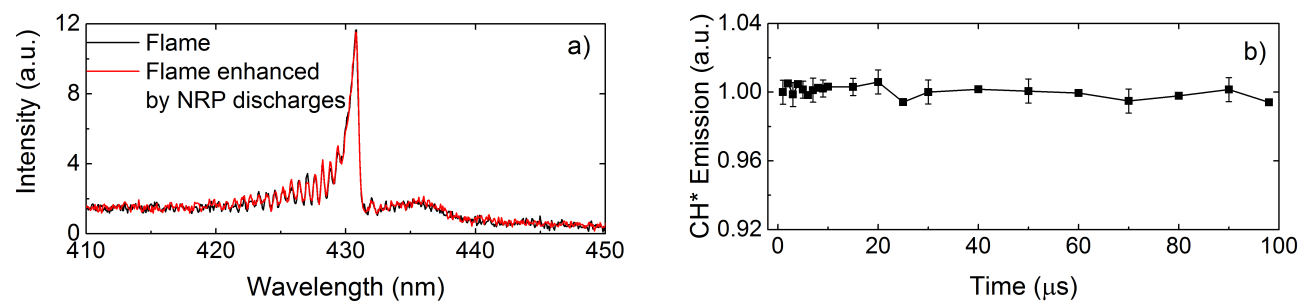

Figure 3: a) Comparison of flame spectrum with flame emission between two pulses of NRP glow discharges, b) Temporal evolution of the global $\mathrm{CH}^{*}$ emission of an M-flame between two plasma pulses.

The step response of the flame to plasma forcing is studied, assuming that the flame is only affected by the averaged electrical power deposited by the plasma, as shown in Lacoste et al. [19], for NRP spark discharges applied at a repetition rate varying from 10 to $80 \mathrm{kHz}$. In the present study, this assumption has been validated by measuring the temporal evolution of the $\mathrm{CH}^{*}$-chemiluminescence of a flame during plasma forcing between two plasma pulses, see Fig. 3b. The averaged $\mathrm{CH}^{*}$ emission of the combustion zone is almost constant between pulses, showing that the flame does not respond to each nanosecond discharge, for the present repetition rate of $10 \mathrm{kHz}$. 
Thus, the step response of the flame to positive and negative plasma forcing, referred to in the following as 'positive step' and 'negative step' of plasma, is investigated by recording phase-locked images of the $\mathrm{CH}^{*}$ emission. The reference time, $t_{0}$, corresponds to the beginning of the first high voltage pulse for a positive step, or to the end of the last high voltage pulse, for a negative step (see Fig. 4). In order to reach a steady-state regime of the flame, negative steps have been investigated after applying NRP discharges during at least $1 \mathrm{~s}$, and positive steps have been studied after at least $2 \mathrm{~s}$ of non-enhanced combustion. The difference between these two values can be explained by the thermalization of the rod (faster heating than cooling process).

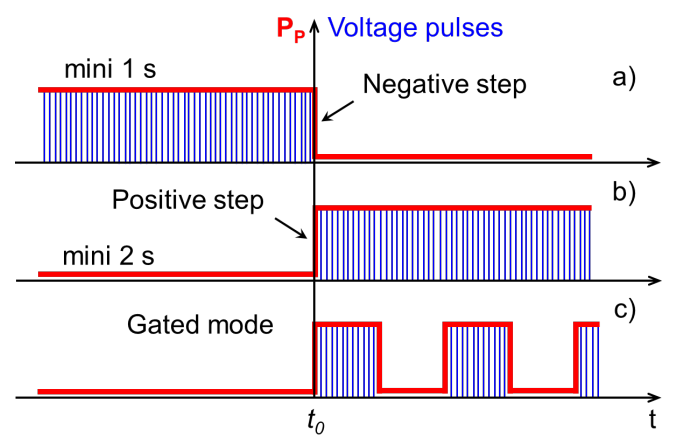

Figure 4: Diagram of forcing sequences: forcing by a) a negative step, b) a positive step, and c) a gated plasma, with a duty cycle of $50 \%$.

\section{HRR fluctuations}

Figure 5 presents the temporal evolution of the HRR of an M-flame, subjected to positive plasma forcing of $1 \mathrm{~W}$ (black squares), and $2.2 \mathrm{~W}$ (red circles), and of a negative plasma forcing of $2.2 \mathrm{~W}$ (blue triangles). First, 
the response of the flame is relatively similar for positive and negative steps of 2.2-W plasma, i.e., with a HRR peak of same intensity (about $6 \%$ of the mean HRR), and similar duration (about $3 \mathrm{~ms}$, defined as the full width half maximum (FWHM) of the peak). It can also be noticed that the HRR does not return to its initial value after applying the plasma steps, probably due to the energy deposition by the plasma, of about $2 \%$ of the HRR. When the plasma power is reduced to $1 \mathrm{~W}$, the HRR peak duration remains the same, but its amplitude is reduced to $3.5 \%$ of the HRR. After $100 \mathrm{~ms}$, its level is similar to that before the plasma step. These results show that the temporal response of the flame is similar for the three different steps, but its amplitude depends on the plasma power.

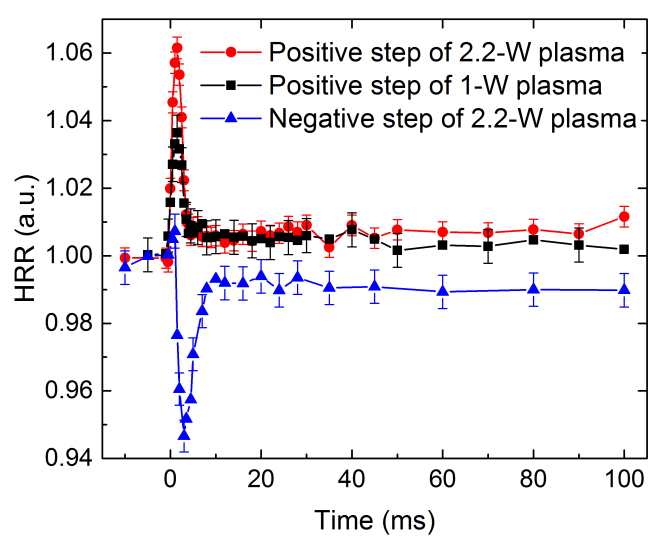

Figure 5: Influence of the plasma power on the response of an M-flame to positive and negative steps of plasma.

Figure 6 compares the HRR temporal evolution of an M-flame subjected to a positive step of 2.2-W plasma (red circles), with that of a V-flame submitted to a positive step of $1.7-\mathrm{W}$ plasma (green triangles). First, the amplitude of the HRR peak is about $20 \%$ higher for the V-flame, compared 


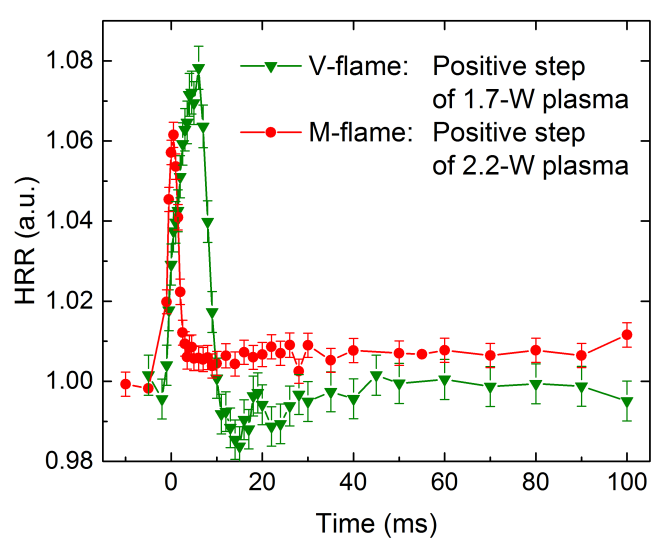

Figure 6: Influence of the flame shape on the HRR response to a positive step of plasma.

to the M-flame, even though the forcing intensity was $25 \%$ lower. Thus, the V-flame is more responsive to plasma forcing. Second, the duration of the HRR peak obtained for the $\mathrm{V}$-flame, about $9 \mathrm{~ms}$, is three times longer than for the M-flame. It can then be concluded that the geometry of the flame affects the HRR response.

\section{Transfer functions}

As plasma formation is a highly nonlinear function of the input voltage, harmonic forcing cannot be realized. However, for small forcing amplitudes, the flame response can be considered as a linear time-invariant system. The transfer function can then be determined from an arbitrary input signal with sufficient frequency content in the relevant range. In order to validate the use of the step response for determining the FTF, two methods of forcing were compared: (i) a step forcing, and (ii) a gated mode with $50 \%$ duty cycle, corresponding to a square-wave modulation of the high-frequency pulse-train. 


\subsection{Definitions}

From the step response of the flame to plasma forcing, method (i), the FTF is calculated as follows. The time derivative of the step response of a linear time-invariant system corresponds to the impulse response, where the integral over the impulse is equal to the step amplitude. Since the Fourier transform of the impulse response is identical to the transfer function, the latter, denoted $F_{P_{i}}$, can be simply obtained by taking a Fourier transform of the time derivative of the step response, normalized with the step height:

$$
F_{P_{i}}(\omega)=\mathcal{F}\left\{\frac{\mathrm{d} Q_{\text {step }}(t) / \bar{Q}}{\mathrm{~d} t}\right\} \frac{\bar{Q}}{P_{p}},
$$

where $\mathcal{F}$ denotes the Fourier transform of a quantity, $Q_{\text {step }}(t)$ is the temporal evolution of the HRR, $\bar{Q}$ is the average HRR of the flame, and $P_{p}$ is the average plasma power.

In the case of square-wave modulation with $50 \%$ duty cycle, method (ii), the amplitude of the fundamental frequency component is related to the square-wave amplitude by a factor of sinc(1/2), which follows from a Fourier expansion of the square-wave signal. If the response of the HRR is only evaluated at the fundamental frequency of the square wave, this factor must be included in the evaluation of the transfer function to plasma forcing, which can be expressed as:

$$
F_{P_{i i}}(\omega)=\frac{\mathcal{F}\{Q(\omega) / \bar{Q})\}}{\operatorname{sinc}(1 / 2) \cdot P_{P}(\omega) / \bar{Q}}=G_{P}(\omega) \mathrm{e}^{\mathrm{i} \phi_{P}(\omega)},
$$

where $Q(\omega)$ is the temporal evolution of the HRR during forcing at the angular frequency, $\omega$, and $P_{P}$ is the averaged plasma power deposited during half a period of the forcing. The transfer function can also be expressed in 
terms of a gain, $G_{P}$, reflecting the level of the flame response, and a phase, $\phi_{P}$, representing a lag between the HRR fluctuation and the middle of each half-period of the forcing. Square-wave modulation is applied for frequencies varying from 36 to $288 \mathrm{~Hz}$, for both V- and M-flames. For each forcing frequency studied, 16 images, phase-locked with the forcing signal, have been recorded, each image being an average of 200 events. Examples of V- and M-flame motions induced by a plasma forcing at $80 \mathrm{~Hz}$ are presented in two movies realized from the $16 \mathrm{CH}^{*}$ chemiluminescence images, see Figs. S1 and S2 of the supplementary material.

The FTFs obtained for M- and V-flames with the two methods are compared in Figs. 7 and 8, respectively (solid lines and scatters). Even with some quantitative discrepancy, the FTFs are in relatively good agreement: the slopes of the phases, and the shapes and amplitudes of the gains are similar. These results show that the step response approach, method (i), can be used for the determination of FTFs for a forcing by non-thermal plasma generated by NRP discharges. However, further investigation will be necessary to understand the differences obtained for the two methods of determining the FTF, especially concerning the maximum gain amplitude, with a discrepancy up to $40 \%$ for the V-flame.

\subsection{Effect of the forcing amplitude on the FTF}

Figure 7 presents the effect of plasma power on the transfer functions of M-flames. At frequencies lower than $350 \mathrm{~Hz}$, the phase of the FTFs is nearly linear and not significantly affected by the plasma power. This is consistent with what is observed in Fig. 5, where the FWHMs of the HRR peaks are similar for the two forcing amplitudes. For frequencies higher than $350 \mathrm{~Hz}$, 
the phase for the 1-W plasma forcing goes up very rapidly. However, since the gain simultaneously reaches a very low value, this could be related to experimental uncertainty.
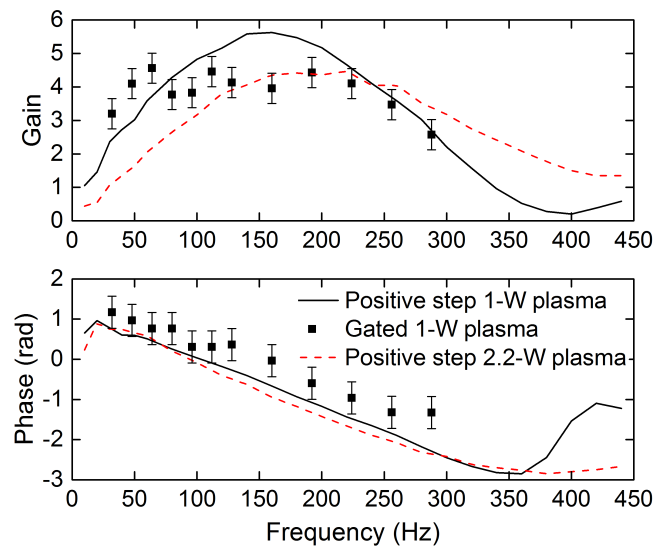

Figure 7: FTFs of M-flames subjected to a positive step of 1-W plasma, a positive step of 2.2-W plasma, and gated NRP discharges at various forcing frequencies.

The gain is slightly affected by the plasma power. First, the maximum amplitude of the gain is about $20 \%$ higher for plasma forcing with the lowest amplitude. Second, the gain shape is globally shifted to higher frequencies when the forcing amplitude is increased. These results are similar to those obtained for acoustic forcing. It is well known that the transfer function magnitude decreases with increasing amplitude of the acoustic forcing [20, 21]. In addition, Schuller et al. [22] have shown that, in the case of transfer functions of premixed laminar flames subjected to flow perturbations, the flame dynamics is governed by two parameters: the ratio of the burning velocity to the mean flow velocity, and a reduced frequency, $\omega^{*}$, defined as:

$$
\omega^{*}=\frac{\omega R}{S_{L} \cos (\alpha)}
$$


where $R$ is a characteristic dimension of the flame, $S_{L}$ the laminar burning velocity, and $\alpha$ the half angle at the top of the flame. This reduced frequency corresponds to the flame Strouhal number, $S t_{f}$, defined by Lieuwen in [23].

In this study, forcing by plasma increases the power of the reactive front by up to $2 \%$ (calculated from $2.2 \mathrm{~W}$ of electrical power deposited by the plasma, and $113 \mathrm{~W}$ of flame thermal power). Without any assumptions about the energy deposition pathway, this additional energy source will lead to a global increase of the burning velocity of the flame. Therefore, as the plasma power is increased, $\omega^{*}$ is expected to shift to higher frequencies. When the plasma power of the forcing is increased from 1 to $2.2 \mathrm{~W}$, the shift of the gain maximum is $50 \mathrm{~Hz}$ (between 150 and $200 \mathrm{~Hz}$, see Fig. 7). Taking into account the experimental conditions, this shift would correspond to an increase in global burning velocity of about $30 \%$. This value is in the range of that which could be expected. As a comparison, if the the fresh gases were heated by a thermal source of $2 \mathrm{~W}$, the increase of temperature would be about $40 \mathrm{~K}$, corresponding to $25 \%$ increase of $S_{L}$. Therefore the reduced frequency $\omega^{*}$ likely remains a relevant parameter for the flame dynamics induced by plasma forcing.

\subsection{Transfer functions of $V$ - and $M$-flames}

To assess whether the reduced frequency is a relevant parameter for plasma forcing, transfer functions of V-and M-flames as a function of $\omega^{*}$ are compared in Fig. 8. The transfer functions obtained for V-and M-flames were determined for an amplitude of plasma forcing of 1.7 and $2.2 \mathrm{~W}$, respectively. These values are not identical but close, therefore in the calculation of $\omega^{*}$, it was assumed that their effect on the burning velocity of the flame 
is linear.
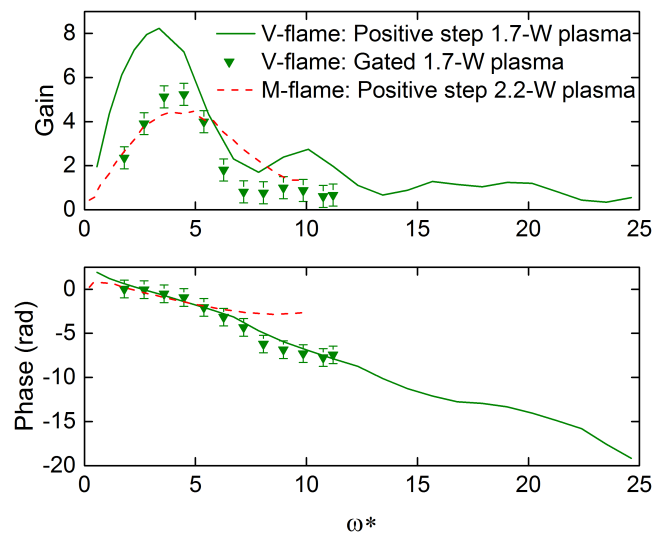

Figure 8: FTFs of V-flames subjected to a positive step of 1.7-W plasma and forced with gated NRP discharges at various frequencies, and FTF of an M-flame subjected to a positive step of $2.2-\mathrm{W}$ plasma.

In Fig. 8, the phases of FTFs obtained for V-and M-flames are very similar at $\omega^{*}$ lower than six, while for higher $\omega^{*}$, the response is slower for the V-flame compared to the M-flame. The gain of the V-flame is higher than for the M-flame, but their shapes are similar, with a maximum obtained for an $\omega^{*}$ of about 4 .

Compared to the FTF obtained for V-and M-flames by Durox et al. [20], these results are similar to those obtained for acoustic forcing, with a forcing amplitude of $6.6 \%$, i.e., in the linear regime of the flame response. The main difference is that for plasma forcing the gain maxima are obtained at $\omega^{*}=4$, while for acoustic forcing the maximum gains were at $\omega^{*}=10$. Further investigation will be necessary to determine the main reason for this shift. However, considering that the flame response to plasma forcing is driven by an increase in the burning velocity, it is not surprising that the 
transfer function has similar characteristics to that corresponding to acoustic forcing. For both forcing types, the flame response originates from purely kinematic mechanisms. For a stationary premixed flame, advection by the flow and upstream propagation of the reactive front balance in each point. While acoustic forcing perturbs the flame surface through advection, plasma forcing acts on the propagation speed, which has a magnitude comparable to the flow velocity. The characteristic time scale for both mechanisms is therefore given by the ratio of the flame height to the bulk velocity in both cases. Consequently, $\omega^{*}$ should also be a relevant parameter for the flame response to plasma forcing.

\section{Discussion}

When forcing by plasma is applied, the geometry of the flame changes, inducing variations in the HRR. In order to assess whether the main action of the plasma forcing could be attributed to a local increase in the reactivity of the flame close to the rod, the temporal evolution of an M-flame forced by a positive step of $1-\mathrm{W}$ plasma has been simulated and compared with corresponding $\mathrm{CH}^{*}$-chemiluminescence images acquired experimentally.

\subsection{Numerical model}

Kinematically perturbed flames can be modeled based on the $G$-equation [24]:

$$
\frac{\partial G}{\partial t}+\mathbf{v} \cdot \nabla G=S_{F}|\nabla G|,
$$

where $\mathbf{v}$ is the flow velocity and $S_{F}$ is the burning velocity. $G$ represents a scalar field whose isosurface $G=0$ corresponds to the flame front. According to Eq. (4), the flame is advected by the flow and propagates normal to itself 
with a velocity $S_{F}$. This model has been successfully used for the determination of transfer functions of elementary, acoustically perturbed laminar premixed flames [22]. Because of the small size of the flame investigated in the present work, curvature effects are significant (see Fig. 2), and the burning velocity must be modified accordingly. This is modeled as $S_{F}=S_{L}(1-\mathcal{L} \kappa)$, where $\mathcal{L}$ is the Markstein length, and $\kappa=-\nabla \cdot(\nabla G /|\nabla G|)$ is the local curvature of the front. The $G$-equation (4) was solved numerically using a 2nd-order TVD Runge-Kutta scheme [25], for time integration, and a 5thorder WENO scheme for spatial discretization [26], in combination with a standard reinitialization procedure [27].

To model the effect of plasma forcing on the flame, we consider a local increase in the reactivity of the flame, altering the burning velocity without affecting the flow field. The impact of the NRP glow discharges on the local $S_{L}$ is expressed as:

$$
S_{L, \mathrm{NRP}}=\left[S_{L}\left(1+30 \% \operatorname{step}\left(t-t_{0}\right)\right)\right], r<1.5 \mathrm{~mm}
$$

Here, $\operatorname{step}(t)$ is a smoothed step-function in the form of a hyperbolic tangent with a rise time of $0.3 \mathrm{~ms}$, and $r$ is the radial coordinate with origin at the center of the rod. The magnitude and spatial distribution of the increase in burning velocity resulting from the discharges is estimated based on the average energy deposition and visual inspection of Fig. 2. In this model, the burning velocity is not affected by the plasma for $r>1.5 \mathrm{~mm}$.

To obtain a flame shape in the simulation that agrees well with the one observed experimentally, it is necessary to use a measured velocity field in Eq. (4). Axial and radial components of the flow field in the presence of an M-flame were measured in the center plane by particle image velocimetry 
(PIV). The measurements were made without NRP discharges. Titanium dioxide particles were used, and the spatial resolution was $100 \mu \mathrm{m}$.

\subsection{Flame dynamics}

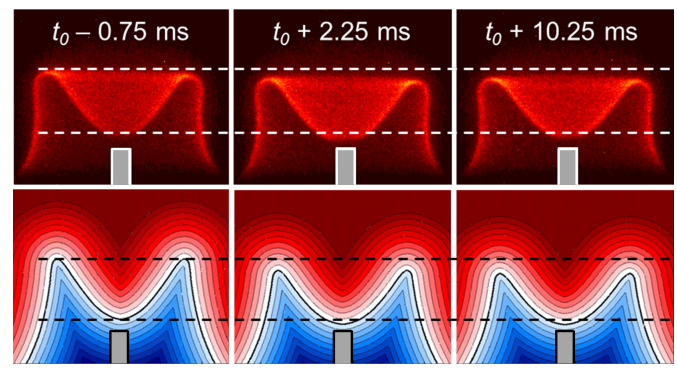

Figure 9: Comparison between the temporal evolution of the $\mathrm{CH}^{*}$ chemiluminescence (upper row), and simulation of the $G$-field (lower row), for a positive step of 1-W plasma, starting at $t_{0}$. In the simulation, the flame front corresponds to the thickened line $G=0$, the fresh gases to the blue area $G<0$, and the burnt gases to the red area $G>0$.

Figure 9 presents $\mathrm{CH}^{*}$ chemiluminescence images and corresponding simulations of the $G$-field of an M-flame forced by a positive step of 1-W plasma. The first column corresponds to the flame prior to the forcing, the second and third columns correspond to the flame 2.25 and $10.25 \mathrm{~ms}$, respectively, after the beginning of the step. The dashed lines correspond to the initial locations of the upper part and the tip of the flame. Both experiments and simulations show a shorter flame after initiation of the plasma, on a time scale as short as $2.25 \mathrm{~ms}$. The middle part of the flame moves upstream, closer to the rod, and the global height of the flame decreases. The experimental and numerical images are in good qualitative agreement. Therefore, forcing by the plasma may be explained by a local increase of the burning 
velocity close to the rod. Further investigation will be necessary to provide quantitative comparison.

\section{Conclusion}

The step response of laminar premixed methane-air flames to non-thermal plasma forcing has been investigated by analyzing the temporal evolution of the HRR when applying positive and negative steps of plasma, generated by NRP glow discharges.

Flame transfer functions were determined from the step response of the flame to plasma forcing. The results obtained are in good agreement with those from a gated plasma at various frequencies.

Similar to the results obtained in the literature for acoustic forcing, the

reduced frequency, $\omega^{*}$, is identified as a key parameter for the flame response to non-thermal plasma forcing.

A local increase in the reactivity of the flame close to the rod appears to be the main mechanism of plasma forcing. Further investigation will be necessary for quantitative comparisons of experimental and simulated FTFs.

\section{Acknowledgements}

This work is partly funded by the Agence Nationale de la Recherche and the German Research Foundation, through the DRACO project (ANR-13IS09-0004), and by the Center of Competitive Funding from King Abdullah University of Science and Technology.

[1] S. Candel, Proc. Combust. Inst. 29 (1) (2002) 1-28. 
[2] T. C. Lieuwen, V. Yang (Eds.), Combustion Instabilities in Gas Turbine Engines, Vol. 210 of Progress in Astronautics and Aeronautics, AIAA, Inc., 2005.

[3] J. Moeck, D. Lacoste, C. Laux, C. Paschereit, in: Proc. 51st AIAA ASM, 2013, paper no. AIAA 2013-0565.

[4] D. Lacoste, J. Moeck, D. Durox, C. Laux, T. Schuller, J. Eng. Gas Turbines Power 135 (2013) 101501.

[5] J. D'Entremont, R. Gejji, P. Venkatesh, S. Bane, in: Proc. 52nd AIAA $A S M, 2014$, paper no. AIAA 2014-0622.

[6] S. Zhang, X. Yu, H. Xiong, H. Zeng, F. Li, Plasma Chem. Plasma Process. 35 (2015) 1029-1042.

[7] W. Kim, J. Snyder, J. Cohen, Proc. Combust. Inst. 35 (3) (2015) 34793486.

[8] A. Sakhrieh, G. Lins, F. Dinkelacker, T. Hammer, A. Leipertz, D. Brantson, Combust. Flame 143 (3) (2005) 313-322.

[9] S. Won, S. Ryu, M. Kim, M. Cha, S. Chung, Combust. Flame 152 (4) (2008) 496-506.

[10] S. Vorontsov, O. Ganeev, P. Tretyakov, A. Tupikin, Combust. Explosion Shock Waves 45 (5) (2009) 530-533.

[11] E. Volkov, V. Kornilov, L. de Goey, Proc. Combust. Inst. 34 (1) (2013) $955-962$. 
[12] J. Kuhl, G. Jovicic, L. Zigan, S. Will, A. Leipertz, Proc. Combust. Inst. 33 (3) (2015) 3521-3528.

[13] Z. Yin, A. Montello, C. Carter, W. Lempert, I. Adamovich, Combust. Flame 160 (9) (2013) 1594-1608.

[14] A. Mazas, B. Fiorina, D. Lacoste, T. Schuller, Combust. Flame 158 (12) (2011) 2428-2440.

[15] D. Rusterholtz, D. Lacoste, G. Stancu, D. Pai, C. Laux, J. Phys. D : Appl. Phys. 46 (2013) 464010 (21pp).

[16] D. Pai, G. Stancu, D. Lacoste, C. Laux, Plasma Sources Sci. Technol. 18 (4) (2009) 045030.

[17] I. Hurle, R. Price, T. Sugden, A. Thomas, Proc. R. Soc. London, Ser. A 303 (1475) (1968) 409-427.

[18] R. Price, I. Hurle, T. Sugden, Symp. (Int.) Combust. 12 (1) (1971) 10931102.

[19] D. Lacoste, D. Xu, J. Moeck, C. Laux, Proc. Combust. Inst. 34 (2) (2013) 3259-3266.

[20] D. Durox, T. Schuller, N. Noiray, S. Candel, Proc. Combust. Inst. 32 (2009) 1391-1398.

[21] P. Palies, D. Durox, T. Schuller, S. Candel, Combust. Flame 158 (2011) 1980-1991. 
[22] T. Schuller, D. Durox, S. Candel, Combust. Flame 134 (1-2) (2003) $21-34$.

[23] T. Lieuwen, Proc. Combust. Inst. 30 (2005) 1725-1732.

[24] A. Kerstein, W. Ashurst, F. Williams, Phys. Rev. A 37 (7) (1988) 27282731.

[25] C.-W. Shu, S. Osher, Journal of Computational Physics 77 (1988) 439471.

[26] R. Fedkiw, B. Merriman, S. Osher, Journal of Computational Physics 157 (2000) 302-326.

[27] D. Peng, B. Merriman, S. Osher, H. Zhao, M. Kang, Journal of Computational Physics 155 (1999) 410-438. 


\section{Figure captions}

Figure 1: Schematic of the experimental setup.

Figure 2: Photographs of the M- and V-flames, a) and c) with NRP glow discharges at $10 \mathrm{kHz}$ and 8-kV applied voltage, b) and d) without plasma.

Figure 3: a) Comparison of flame spectrum with flame emission between two pulses of NRP glow discharges, b) Temporal evolution of the global $\mathrm{CH}^{*}$ emission of an M-flame between two plasma pulses.

Figure 4: Diagram of forcing sequences: forcing by a) a negative step, b) a positive step, and c) a gated plasma, with a duty cycle of $50 \%$.

Figure 5: Influence of the plasma power on the response of an M-flame to positive and negative steps of plasma.

Figure 6: Influence of the flame shape on the HRR response to a positive step of plasma.

Figure 7: FTFs of M-flames subjected to a positive step of 1-W plasma, a positive step of 2.2-W plasma, and gated NRP discharges at various forcing frequencies.

Figure 8: FTFs of V-flames subjected to a positive step of 1.7-W plasma and forced with gated NRP discharges at various frequencies, and FTF of an M-flame subjected to a positive step of $2.2-\mathrm{W}$ plasma.

Figure 9: Comparison between the temporal evolution of the $\mathrm{CH}^{*}$ chemiluminescence (upper row), and simulation of the $G$-field (lower row), for a positive step of $1-\mathrm{W}$ plasma, starting at $t_{0}$. In the simulation, the flame front corresponds to the thickened line $G=0$, the fresh gases to the blue area $G<0$, and the burnt gases to the red area $G>0$. 


\section{Supplemental material}

Figure S1: Movie of a V-flame subjected to a 1.7-W plasma forcing at $80 \mathrm{~Hz}$, realized from $16 \mathrm{CH}^{*}$-chemiluminescence images, taken phase-locked with the forcing signal.

Figure S2: Movie of an M-flame subjected to a 2.2-W plasma forcing at

$80 \mathrm{~Hz}$, realized from $16 \mathrm{CH}^{*}$-chemiluminescence images, taken phase-locked with the forcing signal. 